\title{
XX.
}

Aus der k. k. Universităts-Ohrenklinik in Wien. Vorstand: Hofrat Prof. Dr. A. Politzer.

\section{Beitrag zur Kenntnis der erworbenen Atresien des äufseren Gehörgangs.}

\author{
Von \\ Dr. Rudolf Leidler, \\ Demonstrator der Klinik.
}

Das Bestreben, die durch die Atresie des äußeren Gehörganges bedingten Hörstörungen, sowie manche mit der Gehörgangsatresie verbundene Entstellung des Kopfreliefs operativ zu beseitigen, findet bereits in der alteren Literatur der Ohrenheilkunde dureh zahlreiehe Beispiele ihren Ansdruck.

So mannigfach aber auch die Bemilhungen waren, das Lumen des atresierten Gehörgangs wieder herzustellen, so wenig erfolgreich waren die Eingriffe, die hierzu in Anwendung kamen. Erst in der neneren Zeit sind einige von Erfolg begleitete Operationsmethoden in Anwendung gekommen, welehe als ein wesentlicher Fortsohritt in der operativen Behandlung der Gebörgangsatresien bezeiehnet werden müssen. Mehrere in den letzten Jahren an der Klinik Politzer mit Erfolg operirte Fälle sehienen mir des praktisehen Interesses halber für die Publikation geeignet.

Indem ich im folgenden nur die erworbenen Atresien in den Bereich meiner Betraehtnngen ziehen werde, halte ich es zum Verständnisse der mitgeteilten Fälle für nötig, zunächst die Ätiologie und anatomischen Grundlagen der acquirierten Gebörgangsatresien kurz zu sehildern.

Die Ursachen, durch welche Atresien und hochgradige Stenosen des äußeren Gehörganges hervorgerufen werden können, sind:

1. Unmittelbarer Contakt der von der Epidermis entblößten Gehörgangswände (bei Otit. med. supp. chron.). 
2. Combinierte Caries and Nekrose des Warzenfortsatzes und der Gehörgangswände mit Sequesterbildung und -Ausstoßung.

3. Traumen der verschiedensten Art (vgl. S. 268 und 269).

4. Phlegmonöse, in den Gehörgang sich erstreckende periaurieuläre Entzitindung.

5. Verwachsung massenhafter, von den Wänden des knöchernen Abschnitts ausgehender Granulationen. Hier wäre eine Arbeit Politzers's zu erwähnen, in welcher zwei Fälle von erworbener Gehörgangsatresie beschrieben werden.

Bei dem ersten handelte es sich um allseitige Verwachsung eines gestielten Polypen im äußeren Gehörgange mit den Gehörgangswänden. „Die Nekropsie dieses höchst seltenen Falles ergab eine den ganzen Gehörgang und den oberen Trommelhöhlenraum ausfüllende Bindegewebsmasse, welche von hier in das Vestibulum und den Facialkanal, in den inneren Gehörgang und die Schädelhöhle eindrang, wo sich in der hinteren Schädelgrube mehrere erbsen- bis haselnußgroße Bindegewebsgeschwülste unter der Dura mater vorfanden, welche unter sich und mit den aus dem inneren Gehörgange herauswuchernden Bindegewebsmassen zusammenhingen.

Bei dem zweiten Falle handelte es sich um eine beiderseitige Atresie des knöchernen Gehörgangs durch eine feste fibröse Masse, welche wahrscheinlich infolge einer ehronischen Eiterung dadurch entstand, daß vom Trommelfell und änßeren Gehörgang ausgehende, das Lumen des letzteren vollständig ausfüllende Granulationen untereinander verwachsen sind und in Bindegewebe umgewandelt wurden ${ }^{2}$ ). -

Anatomisch betrachtet, bestehen die Atresien (resp. Stenosen) aus: Bindegewebe oder Knochensubstanz, welehe in verschiedenen Combinationen miteinander vorkommen (vergl. S. 271). Nach außen wird die Atresie von Epidermis überzogen.

Ich gehe nun daran, an der Hand der folgenden Fälle die Methoden darzustellen, welche in den letzten Jahren von $\mathrm{P}_{0}$ litzer zur Heilung von Gehörgangsatresien und -Stenosen verwendet wurden.

I. Atresia meatusauditorii externi sinistri. Ansgeheilte Otitis media suppurativa chronica.

Dr. K, 28 Jahre alt, Arzt aus St. Petersburg, Rußland. Aufgenommen im Juli 1901 .

1) Wiener med. Wochenschr. 1890.

2) Nach Politzers Lehrbuch der Ohrenheilkunde. 4 Aufl. 
Anamnese: Im 4. Lebensjahre Masern, im 17. Typhus. Ein Zusammenhang seines Ohrenleidens mit diesen Erkrankungen ist nicht bekannt. Das rechte $0 \mathrm{br}$ erkrankte $3 \mathrm{mal}$, zuletzt vor mehreren Monaten, an akuter Mittelohreiterung, welche stets austeeilte. An Ohrenfluß im linken Ohre weiß sich Patient nicht zu erinnern. "Seit jeher" hochgradige Schwerhörigkeit auf diesem Ohre. Da sich Patient zufälig studienbalber in Wien aufhielt, consultierte er den Hofrat Politzer, welcher ihm die Operation empfahl.

Status praesens: Patient mäßig gut genäbrt. Innere Organe normal. Im Gebiete des linken Facialis in kleinen Intervallen aufeinanderfolgende tickartige Bewegungen. Andentung von Facialisparese links. Sonst seitens der Himnerven keine Störung. Rechtes Ohr normal

Linkes Ohr. Der Gehörgang endet in einer Tiefe von $c a{ }^{3 / 4} \mathrm{~cm}$ blind. Man sieht am Ende dieses blinden Ganges von unten nach oben einen streifigen, weiBlichen Narbenzug verlaufen. Die Atresie fühlt sich mit der Sonde hart an. Keine Fistelöfnung.

Keine Spontan- oder Druckschmerzhaftigkeit des Warzenfortsatzes. Keine subjektiven Symptome, auBer der hochgradigen Schwerhörigkeit.

Hörweite rechts normal; links wird nur laute Conversationssprache ad concham, durchs Hörrobr auch mäßig laut gehört. Der sonstige Befund entspricht einem hochgradigen Schallleitungshindernisse.

Beim Katheterismus der Tube hört man mit dem Auskultationsschlauche die Luft undeutlich in die Trommelhöble eindringen.

Operation in ruhiger Chloroformnarkose (fecit Professor Politzer). Hautschnitt in der Ansatzlinie der Ohrmuschel. Der Gehörgang wird herausgehebelt. Er setzt sich hinter dem blinden Ende in einen soliden bindegewebigen Strang von einigen Millimetern Länge fort. Nach dessen Entfernung und Abkappung des blinden Endes der bäutigen Atresie sieht man den Gehörgang durch solide Knochenmasse verschlossen. Die Aufmeißelung, welche nunmebr in der Gegend der hinteren oberen Gehörgangswand hegonnen wird, ergibt bis auf $1 / 2 \mathrm{~cm}$ Tiefe nur spongiöse Knochensubstanz. Nach seiner $\mathrm{Abmeilelung}$ erscheint das in seiner hinteren Hälfte perforierte Trommelfell mit dem Hammergriff, und hinter demselben das Stapesamboßgelenk. Die Trommelböhlenschleimhaut trocken, von normaler Farbe. Fs wird nun durch Abmeißelung der hinteren oberen Partien des Gebörgangs ein genügend weites Lumen gebildet, sodann durch radiäre Einschnitte vier Lappen aus dem bäutigen Gehörgangsreste geformt, welche durch Einführung einer Kautschukkanule und teste Tamponade an die Knochenwand allgedrückt werden. Retroauriculäre Wunde durch Nabt verschlossen.

Decursus. 11. Juli 1901. Vollstăndig reaktionsloser, afebriler Verlanf. Erster Verbandwechsel. Die Tampons mit der Kauschukkanüle lassen sich leicht entfernen. Lappen in guter Position. Man sieht jetzt deutlich das Trommelfell mit der ausgedehnten, herzförmigen Perforation und die Gehörknöchelchen.

13. Juli. Die Hörweite hat sich auf $7 \mathrm{~m}$ Konversationssprache nnd $4 \mathrm{~m}$ Flüstersurache gebessert.

24. Juli. Patient wird mit vollständig trockenem epidermisierten Gehórgange in seine Heimat entlassen. Die Horweite hatte sich wieder etwas verschlechtert $13 \mathrm{~m}$, nach Lufteintreibung nach Politzer $5 \mathrm{~m}$ Konversationssprachel. Der Stimmgabelbefund ergab, daB sich das Schallleitungshindernis erheblich vermindert habe.

II. Laesio a uris internae sinistrae, Otitis media suppurativa chronica sinistra. Atresia meatus anditorii externi sinistri.

Leopoldine Scb., 19 Jahre alt, Verkäuferin aus Wien.

Anamnese: Vor 12 Jahren Masern, im Anschlusse daran beiderseitige Otorrhoe. Diese sistierte, und es blieb nur geringe Schwerhörigkeit des afficierten Ohres zurück. Vor ca. 1 Jahre heftiges Stechen im linken Ohre; anschließend daran so rapide Gehörverschlechterung, daß Patientin bald fast gar nichts mehr links hörte und deswegen die Ambulanz unserer Klinik aufsuchte. 
Status praesens rom 24. Februar 1903. Linkes Ohr: Der Gehörgang ist in einer Entfernung von ca. $1 \frac{1 / 2}{\mathrm{~cm}}$ von der äußeren Obröffnung durch eine Haut verschlossen, deren Obertiäche mit der abrigen Gehörgangshaut vollkommen übereinstimmt. Dieselbe ist infolge von Durchschwitzung einer serösen Flüssigkeit immer fencht. Eine ins Innere führende Fistel besteht nicht. Der linke Warzenfortsatz ist druckschmerzhaft.

Rechtes $0 \mathrm{hr}$ : Trommelfell retrahiert, im vorderen unteren sowie im vorderen oberen Quadranten Kalkablagerungen. In den unteren Partien ist die Membrana tympani adhärent. Hinten oben atrophische Narbe. Fieber, Kopfschmerz und Schwindel sind nicht vorhanden.

Linkes Ohr: Konversationssprache und Akumeter ad concham, Flüster. sprache $\theta$; rechts Konversationssprache $\because \mathrm{m}$, Flüstersprache und Akumeter $5 \mathrm{~m}$. Weber nach rechts. Rinne links negativ mit laufgebobener Lutt- und verkürzter Knochenleitung, rechts unentschieden mit gering verkürzter Luftund normaler Knochenleitung. Perception hoher und tiefer Töne links aufgehoben, rechts herabgesetzt. Uhr vom Knochen links und von der stirne negativ, rechts positiv

In Anbetracht der Druckschmerzhaftigkeit am Warzenfortsatze, der eminent schlechten Hörweite und der Möglichkeit einer Eiterung binter der Atresie wurde der Patientin die Operation dringend anemptohlen.

Operation in ruhiger Chlorotormnarkose (fecit Assistent Dr. Alexander) am 24. Februar 1903.

Typischer Hautschnitt. Freilegung des Warzenfortsatzes. Erötinung desselben vom Planum mastoideum ans. Knochen hart, sklerosiert, spröde. In der Tiefe gelangt man zu dem kleinen Antrum, von welchem aus die Radikaloperation in typischer Weise ausgeführt wird. Am Antrum selbst, sowie in der Umgebung desselben ist der Knochen eitrig erweicht. Die Erweichung erstreekt sich bis ins Tegmen tympani, welches teilweise entfernt werden muß, so dalj die Dura der mittleren schädelgrube in ca. Erbsengröße freiliegt. Dieselbe erweist sich normal. In der Trommelhöhle, sowie im Cavum epi- und hypotympanicum Eiter und Granulationen. Der kleine. mißs. bildete Hammer und der Amboß werden entfernt. Vom Hammer erstreckt sich bis an das blinde Gehörgangsende harter sklerosierter Knochen, der den ganzen Querschnitt des Gehörgangs füllt. Derselbe wird bis an den Canalis facialis entfernt, das blinde Ende des häutigen Gehörgangs quer gespalten, und aus dem Gehörgange selbst ein zungenförmiger Plastiklappen (nach $\mathrm{K}$ örner) gebildet, an die hintere Knochenwandilläche angelegt und antamponiert. Die retroauriculäre Wunde wird durch Nähte bis auf den untersten Wundwinkel vollständig geschlossen.

Decursus. Kurze Zeit nacb der Operation regionäre Schmerzen. Dann Woblbefinden Reaktionslose Heilung.

10. März. Patientin wird mit folgendem Status entlassen: Rechts Befund unverändert. Links Wundböhle granulierend. Epithelisierung im Gange. Körnerlappen in situ. Retroauriculäre Wunde bis auf den unteren Winkel primär geschlossen. Hörweite $11 / 2 \mathrm{~m}$ Konversationssprache, Rin ne negativ mit verkürzter Luft- und gering verkurzter Knochenleitung. Perception hoher und tiefer Töne herabgesetzt.

Nach 4 Wochen vollständige Heilung. November 1903 Kontrolle: Heilung anbaltend.

11I. Stenosis meatus auditorii externi et cavi tympanidextri. Otitis media suppurativa chronica bilateralis. Affectionervi acustici bilateralis.

Franz H, 39 Jahre alt, Tagelöhner aus Wien. Aufgenommen am 7. Oktober 1904 .

A namnese: Die Angaben des Patienten sind in Anbetracht seiner geringen Intelligenz sebr wenig brauchbar. Ohrenfluß, Koptschmerzen und starkes Ohrensausen beiderseits, kein Schwindel, kein Fieber. seit 3 Monaten Husten, keine Nachtschweibe

status praesens: Kleines, sehr schwăchliches Individuum. Tuberc. pulmonum. Husten, besonders nachts, wenig Auswurf. Appetit gut. 
Rechtes $\mathrm{Ohr}$ : In einer Tiefe von ca. $2 \mathrm{~cm}$ endet der Gehörgang blind dadurch, dall sich die hintere und obere Gehörgangswand zu einem blindsackartigen Ende nach vorne und unten umbiegt. Vorne unten eine stecknadelkopfgroße Ôffinung, aus welcher dicker, fötider Eiter aspiriert werden kann und auch, wenn auch sehr langsam, spontan hervorkommt. Mit der Sonde fuht sich die Atresie ziemlich hart an. Die Epidermis in der Umgebung der Fistel miffarbig. Warzenfortsatz etwas druckschmerzbaft. Heftige Stirnkoptschmerzen.

Linkes Ohr: Totaldestruktion des Trommelfells. Hammergriffrest an der inneren Paukenwand angewachsen, von stark verdickter Epidermis einseschlossen. Hinten unten schlaffe graurote Granulationen. Die Sonde stöt auf rauhen Knochen. Geringe, ebenfalls fotide Kiterung. Beim Katheterismus der rechten Tube hört man dumpf das Luftgeräusch. Beim kräftigen Durchblasen dringt die Luft mit etwas Eiter durch die Fisteloffinung.

Rechts Konversationssprache ad concham, links $\theta$, mit Hörschlanch undentlich; Flustersprache und Akumeter beiderseits negativ. Weber nach rechrs. Rinne beiderseits negativ. rechts mit stark verkürzter Iuft- und verkürzter Knochenleitung, links mit aufgehobener Luft- und hochgradig verkürzter Knochenleitung, $\mathrm{C}$ negativ, $\mathrm{c}_{4}$ rechts nur stark angeschlagen, links negativ, Uhr vom Knochen negativ.

Operation am 10. Okt. 1904 in ruhiger Chloroformnarkose (fecit Hofrat Politzer). Typischer Hautschnitt. Ablösung des häutigen Gebörgangs. Ausführung der Radikaloperation. Der Knochen ist sehr hart und sklerosiert. Das Antrum liegt sehr tief und ist stark verengt. Der umgebende Knochen desselben erweicht, in demselben Eiter und granrote, schlaffe Granulationen. Bei der Entfernung der binteren Gehörgangswand ergibt sich folgendes: Der knöcherne Gehörgang ist in seiner inneren Hälfte durch concentrische Hyperostose seiner Wände, besonders der vorderen und unteren Wand, stark verengt, so daß es nur ein Lumen von ca. $3 \mathrm{~mm}$ im größten Durchmesser freiläßt, durch welches man in die Trommelböble sieht, ohne jedoch nähere Details darin zu erkennen. Diese Hyperostose setzt sich auf die Begrenzung der Trommelhöhle fort, wo besonders der Boden stark verdickt ist. Es wird nun die hintere Gehörgangswand mitsamt dem bypertrophierten Teile vollständig entfernt, und auch von der vorderen und unteren Wand schichtenweise soviel Knochen entfernt, bis man die Trommelhöhle wie unter normalen Verhältnissen übersieht. Der stark entwickelte Facialiswulst wird geglättet. Das Trommelfell ist vollstăndig destruiert. Hammer und Ambob cariös. In der Trommelhöhle etwas Eiter und Granulationen. Es wird nun nach Abkappung des der knöcbernen Stenose aufliegenden Endes des bäutigen Gebörgangs mit der Schere aus dem ca. $1^{1} / 2 \mathrm{~cm}$ langen Reste ein Körnerscher Plastiklappen geformt und an die hintere Knochenwand in üblicher Weise angelegt. Die retroauriculäre Wunde bis auf den unteren Winkel mittelst Michel scher Klammern primär geschlossen.

Decursus. Reaktionsloser Wundverlauf. Keine Kopfschmerzen. Starker Husten.

18. November. Erster Verbandwechsel. Wundhöhle weit, gut granulierend. Körnerlappen in situ. Retroauriculäre Wunde primär geheilt. Konversationssprache ad concham, Weber nach rechts. Rinne negativ mit stark verkürzter Luft- und Knochenleitung. O negativ, $c_{\&}$ sehr verkürat, Uhr negativ.

Patient steht noch in Behandlung. Die Wundheilung ist durch das Fortschreiten der Tuberc. palmonum verzögert; doch zeigt sich keine Tendenz zur Verengernng.

\section{Otitis med. supp. chron. sinistra. Stenosis meat. audit.} externi.

Franz P., 19 Jahre alt, Knecht aus Mäbren. Aufgenommen am 20. Februar 1905.

An amnese: Seit ca. 3 Jabren Ohrenfluß links, an dessen Beginn sich Patient nicht genauer erinnert Patient hatte hier und da Kopfschmerzen. 
in der linken Kopfhälfte und Schwindel. Da sich diese Symptome in der letzten Zeit verschlimmern, sucht Patient das Spital auf.

btatus praesens: Kräftiges Individuum mit gesunden inneren Organen. Rechtes Ohr normal.

Linkes Ohr: Der Gehörgang, dessen Epidermis normal ist, verengert sich im knöchernen Teile durch Vorspringen der hinteren upd der oberen Gehörgangswand allmäblich $z u$ einer vorne unten gelegenen punktförmigen Öfinung, aus welcher fötider Eiter fließt. Funktionsbefund rechts normal

Links: Weber nach rechts. $1 / 2 \mathrm{~m}$ Konversationssprache; Flustersprache und Akumeter negativ; Rinne negativ mit aufgehobener Luft- und verLarzter Knochenleitung; $\mathrm{C}, c_{4}$ und Uhr vom Knochen negativ.

Keine Druckschmerzhaftigkeit am Warzenfortsatz. Rotatorischer $\mathrm{Ny}$ stagmus beim Blick nach links schwach, beim Blick nach rechts stark. Derzeit keine sonstigen. Schwindelerscheinungen.

Operation am 22. Februar 1915 in ruhiger Chloroformnarkose ffecit Doc. Dr. Alexander): Typischer Hautschnitt. Ausführung der Radikaloperation. Der Knochen ist sebr bart, sklerosiert. Antrum eng; nach hinten von demselben ist ein Teil des Knochens erweicht und von Granulationen durchsetzt. Die hintere knöcherne Gehörgangswand, welche verdickt (insbesondere der Facialiswulst) und von demselben Charakter wie im Warzenfortsatz ist, engt den knöchernen Gebörgang sehr ein. In den übrigen Mittelohrräumen Eiter, Cholesteatommassen und Granulationen. Der cariöse Hammer wird entfernt. Aus dem um die Fisteloffnung nekrotischen Gebörgange wird eine Plastik nach Panse geformt. Wundversorgung, Verband.

Decursus 24. Februar. Derzeit normaler Wundverlanf. Patient befindet sich noch in Spitalsbehandlung.

V. Otitis media suppurativa chronica sinistra. Cholesteatom. Abscessus subperiostalis processus mastoidei. Atresia meatus auditorí externi sinistri.

Innocenz 0, 29 Jahre alt, Tagelöhner aus Holeschovitz in Măhren. Aufgenommen am 3. Oktober 1904.

Anamnese: Seit 24 Jabren linksseitiger Obrenfluß. Ursache unbekannt. Bis vor einem Monat außer starker Schwerbörigkeit keine Beschwerden. Damals bildete sich unter allmählich zunehmenden Schmerzen im Ohre und Warzenfortsatze, sowie in der Stirne und im Hinterbaupte eine Schwellung, binter dem kranken Obre. Fieber, Schüttelfröste oder Schwindel bestanden angeblich nie.

Status praesens: Kräftiger Mann mit normalen inneren Organen. Narbe.

Rechtes Ohr: Trommelfell etwas getrabt, leicht retrahiert, hinten unten

Linkes Ohr: Gehörgang, aus welchem dicker, grünlicher fötider Eiter quillt, durch akute Schwellung der Gehörgangshaut maximal verengt.

Hinter dem linken Ohre, besonders in der Höhe der oberen Hälfte der Ohrmuschel eine starke Schwellung der Weichteile, welche sich nach unten bis auf den Hals, nach vorn bis zum Auge, nach oben und rückwärts weit auf das Kranium fortsetzt. Die größte Höhe $(\mathrm{ca} .3 \mathrm{~cm})$ erreicht dieselbe gleich hinter dem Ohre. Die Haut darüber mäßig gerötet, heiß, wenig gespannt. Deutliche Fluctuation. Die Schwellung ist äußerst spontan und druckschmerzhaft,

Rechts: Konversationssprache über $9 \mathrm{~m}$, Flüstersprache und Akumeter $6 \mathrm{~m}$, links Konversationssprache th $\mathrm{cm}$, Flüstersprache und Akumeter negativ. Weber nach rechts, Rinne rechts positiv mit normaler Luft- und etwas verlängerter Knochenleitung, links wegen der Schwellung nicht zu präten, Luftleitung stark verkürzt. $\mathrm{C}$ and $\mathrm{c}_{4}$ rechts positiv, links stark verkürzt, Uhr yom Knochen von Stirn und rechts positiv, links negativ.

Operation am 5 . Oktober 1904 in rubiger Chloroformnarkose (fecit Docent Dr. Alexander): Hautschnitt über die höchste Convexität der Geschwulst. Es entleeren sich ca. $5 \mathrm{ccm}$ grüngetben dicken Eiters aus einem subperiostalen Abszesse. Im Knochen hinter der Spina supra meatum eine punktförmige Fistelöffnung. Sofort unter der Corticalis erscheint ein Chole- 
steatom, das aus der Knochenöffnung sich hervordrängt. Es ist schmutziggrau, kleinwainußgroß and höchst fötid. Nach Entfernung desselben mit der Matrix ergibt sich eine Höhle, welche das Antrum, den hinteren und oberen Teil des Warzenfortsatzes, den Attik und die Trommelhöhle umfaBt. Hinten liegt der Sinus sigmoideus in einer Länge von ea $1^{1 / 2} \mathrm{~cm}$ frei, seine $W$ and ist verdickt und mit Granulationen besetzt. In der hinteren knöcheruen Gehörgangswand besteht eine Fistel. Nach Entfernung dieser Wand und beim Versuche, die Plastik zu machen, siebt man, daß der membranöse Gehörgang $1 \mathrm{~cm}$ vor dem Trommelfell blindsackartig endet, und von der inneren Fläche dieses Blindsackes sich ein Pfropt von derbem, dichtem Bindegewebe das ganze Gehörgangslumen ausfülend bis zur Trommelhöhle hinzieht. Derselbe wird excidiert, das Ende des Blindsackes mit der Schere abgekappt und aus dem Reste des Gebörgangs eine Plastik nach Panse geformt. Vollendung der Radikaloperation. Die retroauriculäre Wunde wird bis auf den oberen und unteren Wundwinkel offen gelassen

Decursus: 14. Oktober Kopfschmerzen in der linken Schläfe, sonst reaktionsloser Wundverlauf.

20. Oktober. Die Koptschmerzen sind yerschwunden.

28. Oktober. Neuerlich Kopfschmerzen. Temp. 37,8 (leichte Anginal.

29. Dezember. Patient fühlt sich vollkommen wohl. Die retroauriculäre Wunde auf $1 \mathrm{~cm}$ Länge verkleinert. Gebörgang weit. Innere Wunde $\mathrm{ca} .2 \mathrm{~cm}$ tief, größtenteils epitbelisiert. Eine kleine Stelle noch granulierend.

Konversationssprache $3 / 4 \mathrm{~m}$, Flüstersprache und Akumeter negativ. Weber nach rechts, $R$ in ne negativ mit aufgehobener Luft- und verluirzter Knochenleitung. $\mathrm{C}$ negativ, $\mathrm{c}_{4}$ hochgradig verkürzt, Ubr vom Knochen negativ. Patient wird auf seinen Wunsch in seine Heimat entlassen.

\section{Atresia traumatica meatus auditorii externi dextri.}

Sander K. 1), 2 1jähriger Tagelöbner aus Oedenburg in Ungarn. Patient wurde am 24. November 1902 von seinem behandelnden Arzte, Dr. P.2) an die Ohrenklinik gewiesen und daselbst aufgenommen.

A namnese: Arm 30. August 190:3 erhielt Patient einen Säbelhieb über das rechte $\mathrm{Ohr}$ und die rechte Wange, durch welchen das $\mathrm{Ohr}$ an seiner Basis zur Halfte abgetrennt wurde. Die Wunde heilte, doch trat eine Verschlechterung des Hôrvermögens ein. Vorher war Patient nie obrenkrank gewesen.

Status praesens: Ohrmuschelrelief unverändert. An der rechten Gesichtsseite eine $14 \mathrm{~cm}$ lange, lineare, ziemlich gerade verlanfende Narbe, die $3 \mathrm{~cm}$ nach oben hinten rom oberen Enfe der Ohrbasis heginnt und bis gegen das Kinn reicht. Die rechte Ohrmuschel steht ca. $11 / 2 \mathrm{~cm}$ tiefer als die linke. Rechts äulierer Gehörgang vollkommen atresiert. Die Haut der Cymba zieht unverändert daräber hinweg. Linkes Ohr normal.

Hörweite: Rechts Konversationssprache $9 \mathrm{~m}$. Flüstersprache $4 \mathrm{~m}$. links normal. Weber nach rechts, $\mathrm{Scb}$ wa ach verlängert, Rinne recbts negativ mit mäBig verkurzter Luft- und verlängerter Knochenleitung, links positiv und normal. Uhr rom Knochen positiv.

In Anbetracht der relativ guten Hörweite wurde eine membranōse Atresie mit dahinter noch wegsamem Gehörgang angenommen und demgemäß zunächst versucht, die Atresie rom Gehörgange aus abzutragen. Bei der Operation (Operateur Prof. Politzer) zeigte es sicb, daß die Atresie des Gehörgangs eine solide ist, und da man in einer Tiefe von $11 / 2 \mathrm{~cm}$ noch nicht auf Gehörgangsreste stößt, wird nach Abbruch dieser Operation nach 2 Tagen von einem retroauriculären Schnitte wie zur Radikaloperation der vordere Teil des Processus mastoideus freigelegt, die hintere knöcherne Gehörgangswand abgetragen und der atresierende Bindegewebsteil als ca. $2 \mathrm{~cm}$ langer Zapfen gewonnen. Dieser zeigt keinen Hohlraum und wird mit der Schere entfernt. I)ie

1) Vergl. Monatsschrift f. Ohrenheilk. Offic. Protokoll der Sitzung der osterr. otol. Ges. v. 29. Febr. 1404.

2) Siehe Demonstration i. d. Sitzung dieser Gesellschaft am 29. November 1903 . 
gewonnene Gehörgangsöffnung wird nach dem System der Körnerschen Plastik vergrößert, so daß sie für den Nittelfinger durchgängig ist. Bei der Ablösung des Gehörgangsendes quellen einige Tropfen nicht fötiden gelben Eiters aus der knöchernen Gehörgangsöfnung bervor, und nach Applikation eines Katheters wird noch mehr Eiter aus dem Mittelohre entleert. Der knöcherne Gehörgang selbst ist auf die Hältte der normalen Weite verengt. Zur Veckung des fast walnußgroßen Defektes werden $\mathrm{z}$ wei gestielte rechtwinklige Lappen von der Haut über dem Processus mastoideus implantiert; ibre Basis liegt im oberen bezw. unteren Winkel des ersten Hautschnittes Die retroauriculäre Öfnung wird zunächst offen gelassen. Wundversorgung. Verband.

Fünf Tage später werden die beiden Hautlappen an ihrer Basis durchtrennt und die $W$ undränder des retroauricularen schnittes nach duffrischung durch Naht geschlossen Hierauf folgt Deckung des vorderen Teiles der In nochenhöhle durch zwei Thiers chsche Läppchen vom Oberschenkel; ein dritter Epidermislappen deckt die retroauriculäre, von der Lappenbildung herrührende, streifenförmige Wundfläche.

Glatter Verlanf. Fortschreitende Epidermisierung der Höhle. Da in-

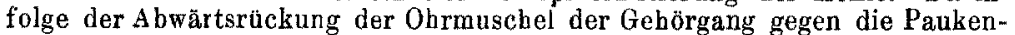
böhle bin steil nach aufwarts verläutt, wird 3 Wochen später ein Stück des Cymbaknorpels exstirpiert und die Gehörgangshaut nach innen gegen den Knochen geschlagen, so daß jetzt auch ohne Speculum die Tiefe des Gehörgangs frei berichtigt werden kann. Eine erbsengroße granulierende Fläche wird gelegentlich einer Thiers chschen Plastik an einem anderen Patienten mit einem von diesem gewonnenen Lpidermislappen, der prompt anheilt, gedeckt.

Hörweite derzeit $5-6 \mathrm{~m}$ Flüstersprache.

Der weitere Wundverlauf war ein vollständig normaler, kein Fieber, keine Schmerzen.

14. März 1904. Patient ver]äßt das Spital mit folgendem Status: Der Gehörgang ist weit, epithelisiert; geringe scbleimig-eitrige Sekretion aus dem* selben. Subjektiv vollstes Wohlbefinden. Hörweite rechts Konversationssprache $9 \mathrm{~m}$, Flustersprache $6 \mathrm{~m}$, Akumeter $3 \mathrm{~m}$. Weber nach rechts, Rinne rechts positiv Hohe und tiefe Töne werden gut gehört, Uhr von Luft und Knochen percipiert.

\section{Atresia meatus a ditorii externi sinistri. Otitis media} suppurativa chronica.

Chaim W., 23 Jahre alt, Schneider aus Russisch Polen. Aufgenommen am 20. Juni 1904 .

Anamese: Vor 2 Jahren Schmerzen im linken Ohre, bald nachher Ohrenfluß. Ursache unbekannt. Der Arzt, den er consultierte, soll ihn mit einer ätzenden Flüssigkeit "gebrannt" haben, jedoch obne Erfolg. Patient wandte sich an einen Ohrenarzt, welcher ihm in längeren Intervallen mehrmals (zuletzt vor ca. 2 Jahren) Incisionen in den äußeren Gehörgang gemacht haben soll, worauf der FluB sistierte. Doch stellte sich ein allmählich bis ins Unertrăgliche sich steigerndes Ohrensausen ein, und die Schwerbörigkeit, welche schon früher in geringem Maße bestanden batte, verschlimmerte sich rapid. Auch traten Kopfschmerzen auf, besonders im linken Scheitel Kein Schwindel, Erbrechen oder Fieber.

Status praesens: Kräftiger Mann mit gesunden inneren Organen.

Rechtes Obr: Trommelfell bis auf geringe Trübungen normal.

Linkes Obr: Der Gebörgang ist in der Tiefe von ca. $1 \frac{1 / 2}{\mathrm{~cm}}$ blindsackartig durch eine von dicker Epidermis bedeckte Gewebsbrucke abgeschlossen. Die Epidermis setzt sich unmittelbar in die Gehörgangswände fort und hat dieselbe Beschaffenbeit wie diese. Das blinde Ende des Gehörgangs fühlt sich mit der sonde hart an.

Linker Warzenfortsatz druckschmerzbaft.

Gehör: Rechts normal.

Links: Konversationssprache $3 \mathrm{~m}$, Flüstersprache $1 / 2 \mathrm{~m}$. Akumeter ad concham. Weber nach links, Rinne rechts positiv und normal, links negativ mit stark verkurzter Luft- und normal langer Knochenleitung. $C$ und 
$c_{4}$ und Uhr rechts normal. Links $\mathrm{C}$ stark, $c_{4}$ gering herabgesetzt. Uhr vom Knochen positiv.

Beim Katheterismus erweist sich die linke Tube stark verengt, aber doch für die Luft durchgängig: nach Katheterismus links geringe Hôrverbesserung $\left(\$^{1 / 2} \mathrm{~m}\right.$ für Konversationssprache, $3 / 4 \mathrm{~m}$ für Flüstersprache)

Operation am 31. Juni 1904 in rubiger Chloroformnarkose (fecit Hofrat Politzeri: Typiscber Hautschnitt. Freilegung und Eröffnung des Warzenfortsatzes. Der Knochen sklerosiert Vereinzelte, bis kirschkerngroße, fötiden Eiter enthaltende Erweichungsherde im Warzenfortsatze, Ausführung der Radikaloperation. Antrum und Paukenbohle von Eiter und Granulationen erfült. Hammer und AmboB nicht vorhanden. Die Atresie endet als băutiger Blindsack. Keine Veränderungen am Knochengerüste des Gehörgangs uvd der Paukenböhle. Es folgt Auskratzung der Tube und Säuberung der Uperationshöble. Nach Durchtrennung des blinden Endes mit dem Skalpell wird die Plastik nach Körner ausgeführt. Tamponade der Wundböhle und des Plastiklappens an die hintere Knochenwundfläche. Vollständiger Schiuß der retroauriculären Wunde mittelst Michelscher Klammern bis auf den unteren Wundwinkel, welcher drainiert witd

Decursus. Reaktionsloser Wundverlanf. Kopfschmerzen verschwunden. Sausen bestebt fort.

18. Juli. Gehörgang und innere Wunde weit; letztere granulierend, teilweise epithelisiert. Geringe Sekretion. Funktionshefund wie oben.

26. Juli. Patient ist eigenmächtig von der Behandlung ausgeblieben. Die Wunde war der vollständigen Heilung nahe. (Nachträglich erfubr ich, dab Patient nach Canada desertiert sei.)

Zum Schlub will ich noch zwei Fälle kurz erwähnen, welche in Bezug auf ihre Ätiologie nicht ohne Interesse sind.

VIll. Otitis media suppurativa chronica bilateralis. Stenosis meatus aditorii externi sinistri post operationem radicalem.

Gisela 0,13 Jahre alt, aus Ungarn. Transferiert von der psychiatrischen Klinik des Prof. Dr v. Wagner-Jauregg am 11. Oktober 1904.

Anamnese: Von der Patientin, welche an Schwachsinn leidet, war nicht mebr zu erfabren, als daß sie seit Jahren an beiderseitigem Ohrenflusse leide und vor einiger Zeit links andernorts operiert wurde.

Status praesens: Linkes $O \mathrm{hr}$ : In der Tiefe von $2 \mathrm{em}$ verengt sich der Gehörgang trichterförmig und läßt endlich nur eine Fistelöffnung frei, aus welcher fôtider Eiter kommt und durch welche man mit der Sonde in eine Höhle eindringt. Am Warzenfortsatz Operationsnarbe. Keine Druckschmerzen.

Rechtes Ohr: Chronische Mittelohreiterung.

Beiderseits nur laute Konversationssprache ad concham. Weber nach links. Die übrige Prüung ergibt beiderseits bochgradiges Schalleitungsbindernis.

25. Oktober. Operation in Chloroformnarkose (fecit Hofrat Prof. P olitzer): Hautschnitt in der alten Operationsnarbe. Im Warzenfortsatz weit gehende eitrige Frweichung bis in die Spitze und knapp an den Sinus. Der wahrscbeinlich neugebildete Facialissporn, ebenso die erkrankte vordere Gehörswand werden teilweise abgetragen, der Attik mit der Wundhöble in ein Niveau gebracht Auskratzung der Trommelhöhle. Die Stenose, welche dadurch entstanden war, daß die Epidermis des Gehörgangs ein (von der Fistel durchlöchertes) Septum von ziemlicher Dicke vor der Wundböhle gebildet hatte, wird excidiert und aus dem Reste des häutigen Gehörgangs unter Zuhilfenahme eines Teiles der Concha eine K örn e r sche Plastik geformt.

Decursus: Reaktionsloser Wundverlauf.

IX. Septumbildung nach Radikaloperation links wegen 0 tit is media suppurativa chronica.

Josef M., 22 Jahre alt, Kellner aus Ungarn. Aufgenommen am 14. OKtober 1904.

Anamnese: Patient wurde bereits 3 mal auswärts wegen chronischer, 
immer recidivierender Eiterung links operiert, zuletzt vor 6 Monaten Jetzt besteht wieder heftiges Stechen in der linken Kopfbälfte, Schwindel und Erbrechen. Kein Fieber. Geringe Eitersekretion.

Status praesens: Schwächliches Individuum mit gesnnden inneren Organen. Augenbefund: Anämischer, sonst normaler Fundus.

Rechtes Obr normal.

Linkes Ohr: Die Operationshöhle ist bis anf eine erbsengroße Stelle oben epithelisiert. Daselbst Granulationen und eine nach hinten oben füh remle ca $3 / 4 \mathrm{~cm}$ lange Fistel. Sondierung schmerzhaft. Kein rauher Knochen füblbar. Hinter dem Ohre drei Operationsnarben. Starker, fast continuierlicher Schwindel mit Erbrechen. Schwanken beim Steben mit geschlossenen Augen, horizontaler Nystagmus beim Blick nach rechts.

Hör- und Funktionsbefund: Rechts annäbernd normal, links laute Konversationssprache ad concham. Weber nach rechts, $\mathrm{C}, \mathrm{c}_{4}$, Uhr vom Knochen negativ, Rinn e negativ mit aufgehobener luft- und verküzter Knochenleitung.

Operation in rubiger Chloroformarkose (fecit Docent Dr. Alexan der) am 23. Oktober 1904: Schnitt in einer Operationsnarbe. Freilegung der alten Radikaloperationshöhle. Die Weichteile sind in ein äuBerst derbes, sehnig glänzendes Narbengewebe umgewandelt, welches gegen die mittlere Schädelgrube hin mit der in ca. Zwanzigbellerstückgröße freiliegenden Dura fest verwachsen ist. Nach Ablösung der hinteren Gehörgangswand vom Knochen bemerkt man, daß der Gehörgang durch ein Epidermisseptum von der dahinter liegenden 'Trommelhöhle getrennt ist. Dieses Septum ist dadurch entstanden, daß die Epidermis der vorderen Gehörgangswand nach hinten umbiegend zugleich mit dem Plastiklappen an dem stark vorspringenden Facialiswulst angewachsen war. In der Trommelhöhle Retention von Eiter und Cholesteatom. Vas Ende des blindsackartigen Septums wird soweit abgekappt, daß wird ein neuer K örnerscher Lappen geformt; Glättung des Facialiswulstes und Ausräumung der Trommelhöhle. Der Knochen überall gesund. Schließung der retroauriculären Wunde mit $\mathrm{Michelscben} \mathrm{Klammern.}$

Decursus: Reaktionsloser Wundverlauf. Patient ist beschwerdefrei.

7 . November. Retroauriculäre $W$ unde primär verheilt.

Innere Wunde granulierend, teilweise epithelisiert. Mäßige Sekretion. Hör- und Funktionsbefund unverändert. Kein Nystagmus. Patient wird auf Wunsch in ărztliche Behandlung in seine Heimat entlassen.

Ehe ich zusammenfassend die oben referierten Fälle näher bespreche, will ich zum besseren Verständnisse besonders der daselbst angewandten Operationsmethoden eine kurze Geschichte der operativen Therapie von erworbenen Atresien geben.

Bonnafont ${ }^{1}$ ) hat als einer der ersten mit einer rattenschwanzähnlichen Feile eine knöeherne Atresie durchbohrt, so dass sie einige Jabre hindurch durehgängig blieb.

A yres ${ }^{2}$ ) benutzte eine Methode, mit welcher schon früher Matterson aus Brooklyn erfolgreioh eine den Gehörgang obturierende Knochenneubildung entfernt batte. Er versuchte nämlich mit einer Zahnbohrmaschine eine vollständige Atresie des Gehörgangs zu beheben. Er gelangte unter großer Mühe, nachdem er teils wegen ungenügender Länge des Bohrers, teils wegen

1) Monatsschr, f. Ohrenb. II. Nr. 8.

2) Zeitschr. f. Ohrenh. XI. 1882. 
schlechter Anästhesierung des Patienten einige Male unterbrechen mubte, durch die Atresie hindurch. Es folgte dann Eiterung aus dem Ohre, welche nach zwei Wochen sistierte. Die Hörweite hatte sich etwas gebessert. Uber eine eventuelle Heilung und die Größe der Öffnung finden sich keine Angaben. Verfasser gibt selbst die Gefabren dieser Methode in eventneller Verletzung des Mittelobres oder sogar des inneren Ohres an und charakterisiert am besten die Brauchbarkeit derselben dadurcb, dab er die Atresie, welehe der Patient im anderen Gehörgange hatte, unangetastet ließ.

Rotbolz ) beseitigte einen membranösen Versehlub des änßeren Gehörgangs nach Otitis media suppurativa chronica durch mehrfache radiäre Incisionen der obturierenden Membran und Einlage von Wattetampons. Die Membran sehwand bis auf einen kaum $1 / 2 \mathrm{~mm}$ breiten Rand und lieb dahinter das Trommelfell mit zwei Perforationen sehen. Die Hörsebärfe war gestiegen, und es bestand keine Tendenz zur Recidive.

In selben Jahre (1885) beschreibt Mandelstamm ${ }^{2}$ ) aus Kiew eine narbige Verwachsung des Ohreinganges mit Otitis med. supp. chron., bei welcher es durch Staung des Eiters im Gehörgang zu einer Erweiterung des letzteren gekommen war. Es fand sich am Ohreingange eine nach außen gewölbte, deutlich fluetuierende narbige Membran, welche den Eingang vollständig verschloß. Mandelstamm machte eine Probepunktion mit einem Troicart und hierauf eine ergiebige Aufschlitzung der Narbenmembran, worauf sich eine große Menge einer seröseitrigen Flüssigkeit entleerte. Der Gehörgang erwies sich besonders unter dem Tragus sackartig erweitert; die Gehörgangswände waren in der Epidermissehicht maceriert. Das Trommelfell war ebenso maceriert und hinten unten perforiert. Auf der Perforation lag etwas Eiter. Nach einer Lufteinblasung nach Politzer besserte sich sofort die Hörweite, die vorher recht sehlecht gewesen war. Da Patient keine Zeit zu einer weiteren Behandlung hatte, wurde ihm ein kurzes, dickes Hörrohr aus Neusilber eingeführt, um einer Verwachsung der Schnittränder vorzubeugen. Nach einem Jahre stellte er sich wieder vor. Er war vollständig geheilt: Der Ohrenfluß verschwunden, die Perforation des Trommelfells geheilt und die Hörweite noch gebes-

1) Zeitschr. f. Ohrenh. XV. 1885.

2) Monatsschr. f. Ohrenh. Bd. XIX. 
sert. Das Röhrchen hatte Patient schon längst abgelegt. Die äußere Öffnung erwies sich weiter als auf der anderen Seite.

Cheatham ${ }^{1}$ ) berichtet über einen Fall von ,sekundärem Trommelfell" (membranöser Verschlub beider Gehörgänge nach chroniseher Eiterung), welches er unangetastet lieb in der Annahme, daß das ehemalige Trommelfell durch die Mittelohreiterung zugrunde gegangen war und in seiner Funktion durch diese nengebildete Membran ersetzt worden sei.

Si eben $\operatorname{man} n^{2}$ ) beschreibt eine narbige Stenose des äußeren Gehörgangs nach einem luetischen Geschwür mit Otitis media supp. chron. und epileptiformen Anfällen. Spaltung des mehrere Millimeter dicken Diaphragmas mit dem Messer und nachfolgende vom Patienten weitergefübrte Bougierung mit hartgewickelten Jodoform-Wattetampons durch 6 Wochen hindurch. Diese Therapit stellte die ehemalige Meatusweite wieder her, die sich seither während 10 Monaten vollständig gut erhalten hat.

Jansen ${ }^{3}$ ) hat im Jahre 1894 zwei Fälle von Narbenstenose des äußeren Gehörgangs nach einem plastischen Verfahren geheilt, welches er besonders bei Stenosen des äußeren Gehörgangs nach luetischen Geschwüren und Traumen zur Anwendung empfiehlt. Es handelt sich um Transplantation gestielter Hautlappen a) aus der Gegend des Warzenfortsatzes auf die hintere Gehörgangswand, und b) aus der hinteren Fläche der Ohrmuschel auf die vordere Gehörgangswand nach Excision des sehwieligen und narbigen Gewebes.

Bei der überwiegenden Mehrzahl dieser Fälle handelte es sich um relativ dünne Membranen, welche den Gehörgang verschlossen, ohne daß es zu einer höhergradigen Verstopfung desselben durch neugebildetes Gewebe gekommen wäre. Daher leiten sich die guten Erfolge durch einfache Methoden her. Wo aber, wie bei den ersten zwei Fällen die Atresie durch Knochenwucherung kompliziert war, erwies sich die Beseitigung derselben vom Gehörgange aus teils als ungenügend, teils als direkt gefährlich. Nun muß man aber nach den Erfahrungen der neueren Zeit annehmen, daß die Zahl dieser letzteren (mit höhergradiger Gewebsneubildung komplizierten) Atresien viel größer war, als es nach der Anzahl der veröffentlichten Fälle erscheinen möehte. $\mathrm{Ja}$, was wenigstens die Atresien infolge chronischer Eiterungen

1) Zeitschr. f. Ohrenheilkunde. 1886.

2) Zeitschr. f. Ohrenheilkunde. XXI, 1891.

3) Arch. f. Ohrenheilkunde. Bd. XXXVII. S. 135.

Archiv f. Ohrenheilkande. LXIV. Bd. 
anbelangt, ist es höchst wahrscheinlich, daß ihre Zahl früher, wo einerseits das Publikum den Ohreneiterungen noeh viel weniger Achtsamkeit entgegenbrachte, andererseits die operativen Radikalbehandlungen derselben viel seltenerer als jetzt geübt wurden, noch viel größer gewesen sein muBte, als jetzt.

Da aber gerade diese Fälle nicht selten grobe subjektive Beschwerden und gefäbrliche Komplikationen infolge von Eiterretentionen und fortschreitender Eiterung hinter der Atresie verursachen, ist es begreiflioh, daß man überall bestrebt war, eine radikale Methode zur Beseitigung derselben zu ersinnen.

Körner') war der erste, weleher 1896 an der Hand eines Falles von narbiger Stenose des Gehörgangs nach einer Verletzung durch Hufsehlag eine Operationsmethode erworbener Gebörgangsversehlüsse angab, die der jetzt geübten Methode bereits nahe kam.

Er operierte die nur für eine dünne Sonde passierbare Stenose folgendermaßen: "Die Ohrmuschel und der knorpelige Gehörgang wurden wie bei der Radikaloperation abgelöst und nach vorn gedrängt. Dann wurde der knöcherne Gehörgang mit dem Meißel nach hinten erweitert und aus der hinteren Gehörgangswand durch zwei parallele Schnitte ein an der Muschel sitzender Lappen gebildet und dieser, nachdem die Muschel wieder angenäht war, nach hinten gegen die Wand des erweiterten knöchernen Gehörgangs antamponiert. Die Heilung war nach drei Wochen vollendet und ein so weites Lumen erzielt, wie es sich durch einfache Spaltung und Excision in solchen Fällen nie erreichen läbt."

Sehr ausfuhrlicb hat sich Sehwartze ${ }^{2}$ ) in Halle mit dieser Frage beschäftigt. Den Kernpunkt seiner Arbeit bildet folgendes:

Schwartze empfiehlt in allen Fälen (mit Eiterung und auf andere Weise entstandenen Fällen) die Operation. Dieselbe gestaltet sich im großen und ganzen folgendermaßen: Er löst den membranösen Gehörgang vollständig $a b$, führt die typische Radikaloperation aus und formiert aus der hinteren, membranösen Gehörgangswand zwei Lappen, die er im oberen und unteren Wundwinkel durch Nähte fixiert.

Selbstverständlich wird diese Operation in den verschiedenen Fällen den jeweiligen Verbältnissen angepaßt, so daß sie manche, aber nicht wesentliche Modifikationen erfährt. Diese Operations-

1) Verhandlgn. d. deutschen otol. Gesellschaft. 1896. S. 105.

2) Arch. f. Ohrenheilk. Bd. XLVII u. XLVIIL. 
methode empfiehlt Schwartze nicht nur bei denjenigen Fällen, wo eine hinter dem Verschlusse des Gehörgangs befindliche Krankheit an und für sich eine Knochenoperation erfordern würde, sondern in jedem Falle von Atresie und bochgradiger Stenose.

Er begründet diese Therapie durch die sehr ausfübrliche Schilderung von 12 von $\mathrm{ihm}$ auf diese Weise behandelten und jahrelang beobachteten Krankheitsfällen. Bei den 12 Fällen handelte es sich 7 mal um hochgradige Striktur und 5 mal um vollständige Atresien. Die Verengerung war 4 mal häutiger, $7 \mathrm{mal}$ häutiger und knöcherner und 1 mal unbekannter (wegen Verweigerung der Operation) Natur. Was die Ursachen anbelangt, so machte Schwartze die Beobacbtung, daB bei 11 Fällen der Atresie ein Trauma (im weiteren Sinne) zugrunde lag, während nur ein Fall spontan durch Otit. med. supp. chron. entstanden war. Und zwar handelte es sich in der Mehrzahl der Fälle um operative Traumen (7 Fälle sicher und 2 möglich durch Mastoidoperation und andere operative Eingriffe, welche die bei allen diesen Fällen nebenbei bestehende Otit. med. supp. chron. erfordert hatte).

Die hänfig gefundene Hyperostose faßt Schwartze entweder als disponierendes Moment oder als mittelbare Folge des Traumas auf.

Neunmal wurde Totalaufmeißelung und Strikturoperation gemacht, und nur 2 mal entfiel die Knochenoperation aus äueren Grïnden. Bei diesen beiden Fällen trat Wiederverengerung ein.

In keinem Falle bestand trotz der teilweise vollständigen Eiterretention eine intracranielle Komplikation.

Was die Heilerfolge anbelangt, so kam er zu folgenden Resultaten: $7 \mathrm{mal}$ ist die Eiterung geheilt, bei 2 Fallen erfolgte keine Heilung und ein Fall blieb unbekannt. Die Atresie, resp. Stenose wurde $7 \mathrm{mal}$ geheilt. Einmal trat rollständiges, dreimal partielles Recidiv ein.

Die Erfolge waren also im Vergleich zu den Erfolgen mit den früheren Methoden sehr zufriedenstellend. "Trotzdem bietet", wie Schwartze selbst zugibt, "selbst diese Methode nicht immer Schutz gegen Reeidive, welche noch nach Jahren eintreten können. Es ist eben eine Neigung zur Wiederverengung da, welche auf unbekannter Ursache beruht". "Vielleicht ist", so meint Schwartze, "diese Neigung zur Knochenhypertrophie eine Folge chronischer Haut- und Periostreizung, herbeigefuhrt durch 
Stagnation des Sekretes dahinter. Dieselbe Neigung zu Recidiven beobachtete Sehwartze anch bei einfacher Hautnarbenstriktur ohne Knochenhypertrophie. Doch meint Sehwartze wohl mit Recht, dab selbst ein Recidiv der Atresie den Patienten nioht mehr gefährden kann, wenn nur die Eiterung ausgeheilt ist.

Von den folgenden Arbeiten iber dieses Thema sei nur noch

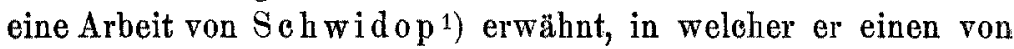
ihm vor Erscheinen der Schwartzeschen Arbeit mit gutem Erfolge operierten Fall von totaler narbiger Atresie infolge eines Traumas schildert. Die Operationsmethode gleicht im wesentlichen der von $\mathrm{K} \ddot{\mathrm{rn}} \mathrm{er}$ (siehe oben) angegebenen, nur dab Schwidop Panses Plastik und auBerdem auch noch zwei Lappen in der Ohrmuschel machte und alle vier Lappen teils annähte (Gehörgangslappen), teils tamponierte (Muschellappen), eine Plastik, die wegen der großen Gefahr einer Perichondritis nicht zu empfehlen ist. Wegen Neigung zur Wiederverengerung mubte dem Patienten eine Prothese gegeben werden, welche er durch 4 Jahre hindurch ohne Beschwerden trug. Verfasser empfiehlt ferner überall dort, wo die häutige Gehörgangswand vom Knochen abgehoben wurde, behufs festerer Verwachsung des Gehörgangs mit dem Knochen die Corticalis in feinen Schichten abzutragen.

Hierher gehört auch eine Arbeit von $R u^{2} d_{0} \operatorname{lphy}^{2}$ ) in Breslau, "Über traumatische Gehörgangsatresien". In derselben referiert der Verfasser ủber die einschlägige Literatur an der Hand folgender Einteilung solcher Atresien resp. Strikturen:

1. durch rohe Gewalt auf den Gehörgang und die Ohrmuschel entstandene (Hufschlag, Verletzung bei Zangenoperationen und dergl.), bei welchen es nur bei unvollständiger Abreibung zu Verengerungen kommt, bei vollständiger nieht: Courtade, Schwidop, Raoult, Körner, Sehwartze;

2. durch Schubverletzungen: Corradi, Reinhard, Politzer, Ferrer;

3. dureh schlecht ausgefuhrte Mastoidoperationen: Sehwartze;

4. durch schlecht verheilte Fraktur des äußeren Gehörgang:s (selten!). Nach Haßlauer entstehen sie: a) direkt durch Gewalteinwirkung auf die Kopfseite (Fall, Stoß, Schlag); b) indirekt, a) durch Stoß des Unterkieferköpfehens gegen die vordere Ge-

1) Arch. f. Ohrenheilkunde. Bd. LIII. 1901.

2) Zeitschr. f. Ohrenheilkunde. 1903. 
hörgangswand bei Fall auf das Kinn oder heftigen Schlag gegen das Kinn; $\beta$ ) durch Fall auf das Hinterhaupt durch Contrecoup. In letztere Gruppe gehört ein Fall von Schwartze und ein Fall vom Verfasser selbst, bei welchem es sich um eine doppelseitige Gehörgangsverletzung durch Anprall des Unterkiefers gegen die vordere Wand, besonders links, infolge eines Hufschlages handelte. Rechts erfolgte Heilung; links Fraktur der vorderen Gehörgangswand vielleicht mit Abreißung des knorpeligen vom knöchernen Gehörgange mit nachfolgender Narbenatresie. Die Operation wurde vom Patienten abgelehnt. Verfasser gibt dann einige Winke für die Behandlung von Gehörgangsverletzungen: Bei frischen Fällen soll man neben der Antisepsis auch auf die Verhütung von Stenosen bedacht sein (am besten durch Einschieben fest gedrehter Wattebäusche). Bei bereits eingetretener Verengerung rät Verfasser zur Operation ${ }^{1}$ ), und zwar empfiehlt er als Methode die schon von Körner, Schwartze und Schwidop angegebene: Schnitt hinter der Ohrmuschel, Ablösung des häutigen Gehörgangs, Erweiterung des knöchernen Gehörgangs durch Abmeißelung concentriseher Knochenlamellen und Plastik nach $\mathrm{Körner}$ und Panse. Unbedingt ist die Beseitigung der Stenose nur in solehen Fällen anzustreben, wo Eiterung besteht. Sonst ist die Operation zur Hörverbesserung angezeigt, wenn der schallpereipierende Apparat nicht zu sehr geschädigt ist.

Der Vollständigkeit halber will ich zum Schlusse noch einige Worte über die Therapie der congenitalen Atresien des äußeren Gehörgangs sagen.

Diese Form von Atresien wird durch Mißbildangen, zumeist Entwicklungshemmungen im Bereiche der ersten Kiemenspalte erzeugt. Rudimentäre Fntwicklung oder Totaldefekt der Ohrmuschel, nicht selten mit Mißbildungen im Bereiche des Mittelohres verbunden, wurden in der Literatur zumeist als Teilbefunde bei der Beschreibung von Mißbildungen des Gehörgangs verzeichnet. Da bei den Mißbildungen des Gehörorganes das Labyrinth verhältnismäbig häufig intakt gefunden wird - (es besteht in einer ziemlichen Anzahl der Fälle trotz der Atresie ein leidliches Hörvermōgen, in den anderen Fällen läßt sich durch funktionelle Untersuchung die Erkrankung des Labyrinths nachweisen; zumeist sind diese Fälle mit Verödung des Carum tympani

1) Nebenbei erwähnt er einen Fall von traumatischer Stenose, welche von 0 stmann in Berlin durch Elektrolyse geheilt wurde. 
und Defekt der Entwicklung der Gebörknöchelchen combiniert; auch subjektive Beschwerden [Kopfschmerzen, Schwindel usw.] sind meist nicht vorhanden), - da auferdem nach den in der Literatur bekannten Fällen von congenitaler Gehörgangsatresie, bei welcher die Operation unternommen wurde, durchaus klägliche Resultate die Folge waren, können wir den Satz aufstellen, daß die congenitalen Gehörgangsatresien nur in Ausnahmefällen Objekte eines chirurgischen Eingriffes sein können. Ein operativer Erfolg ist gewöhnlich dadurch ausgeschlossen, daß in der Mehrzahl der Fälle außer dem membranösen Gehörgange auch noch der knöcherne vollständig fehlt.

Das Trommelfell fehlt gänzlich oder ist rudimentär vorhanden und nach außen von einer dicken Compacta gedeckt. Da sich nun an den Warzenfortsatz direkt die Fossa artionlaris mit dem Unterkieferköpfehen ansetzt, ist auch nicht Aussicht vorhanden (was auch sonst technisch schwierig und funktionell bedeutungslos wäre), einen künstlichen Gehörgang zu bilden. Für seltene Fälle, in welchen der Gehörgang nur durch ein membranöses Septum rerschlossen ist, könnte ja immerhin die Operation versucht werden. Politzer beschreibt in seinem Buche diese Fälle und gibt auch an, daß diagnostisch die Deutlichkeit, mit welcher man beim Katheterismus trotz der Atresie die Luft durch die Tube hindurchstreichen hört, verwertet werden kann.

Überblicken wir nun zusammenfassend die oben beschriebenen, an der Universitäts-Ohrenklinik in Wien operierten Fälle, so ergibt sich folgendes:

Unter den 8 Fällen handelte es sich 6 mal um Atresien und 2 mal um hochgradige Stenosen (III, VII) des äußeren Gehörgangs.

Die Anamnese war in allen Fällen unzutreffend, indem in keinem einzigen Falle der Patient von der Existenz seiner Atresie wußte. Die hauptsächlichsten Klagen, mit welchen die Patienten in die Klinik kamen, waren: Schwerhörigkeit (9 mal), Kopfschmerzen (5 mal), Ohrensausen (4 mal), Ohrenflub (3 mal), je einmal Schwellung hinter dem Ohre und Erbreehen, zweimal Sehwindel.

Als $\ddot{A}$ tiologi e ergab sich Otitis media suppurativa chronica in allen Fällen außer einem (V); Trauma in 4 Fällen und zwar: Fall V: Säbelhieb mit unvollständiger Abreißung der Obrmuschel (vergl. Rudolphys Arbeit, S. 32, 1). 
Beitrag zur Kenntnis der erworbenen Atresien des äuBeren Gebörgangs. 271

Fall VI. Häufige Ätzungen und Incisionen im änßeren Gehörgang; Fall VII und VIII Radikaloperation.

Zur Verhütung der Atresie nach Radikaloperation sind folgende Punkte besonders zu berïcksichtigen: 1. möglichst weitgehende Abtragung des Facialiswulstes; 2. Bildung einer Wundhöhle mit glatten Wänden ohne Buchten und Leisten; 3. Bildung einer genuigend großen Gehörgangsöffnung bei der Plastik; 4. sorgfältige Kontrolle bei der Naehbehandlung, welche besonders auf eine Verengerung des Gehörgangs und auf eine Septumbildung vor der Wundhöhle Bedacht nimmt.

In Bezug auf die anatomische Beschaffenheit wurde der Abschluf des Gehörgangs gebildet: durch Epidermis 3 mal durch Epidermis und Bindegewebe 2 mal, durch Epidermis und Knochen 2 mal, dureh Epidermis und Bindegewebe und Knochen 1 mal. Bei den knöehernen Atresien konnte man zwei Arten unterscheiden: 1. Versebluß durch spongiösen Knochen (I), weleher sieh, wenigstens teilweise, anf der Basis von Bindegewebe gebildet haben dürfte; 2. durch sklerosierten Knochen (II, III, IV). Da bei den letzteren Fällen alle übrigen Mittelohrräume (besonders Antrum und bei Fall III auch Trommelhöhle), und Warzenfortsatzräume (Zellen) dureh sklerosierten Knochen stark eingeengt waren, so erscheint die Annahme berechtigt, diesen Versebluß (resp. Veren. gerung) des Gehörgangs als eine Teilerseheinung einer allgemeinen Hyperplasie des Knochens dergesamten Gehörorganskapsel anzusehen, infolge welcher der Knochen sowohl verdichtet wird als auch die Hohlränme concentrisch verengt werden.

Die Therapie war in 7 Fällen dureh die Symptome der ehronischen Otitis vorgezeichnet, und das Bestehen der Atresie (resp. Stenose) bildete nur eine Indikation mehr, die Radikaloperation auszufuhren. Nur bei 2 Fällen bildete die Atresie als solehe die Indikation zur Operation, welche in beiden Fällen den gegebenen Verhältnissen entsprechend gestaltet wurde. Im Falle I wurde die begonnene Radikaloperation nicht ganz durohgefthrrt, da die Otitis vollständig ausgeheilt war; man begnügte sich mit der Entfernung der Atresie. Zur Gehörgangsplastik wurde überall dort, wo ein genügend langer Gehörgangsrest zur Verfügung stand, die Körnersche Methode verwendet. Sonst 
272 XX. LEIDLER, Beitrag zur Kenntnis der erworbenen Atresien usw. wurden teils specielle Plastikmethoden (I, V), teils die Plastik nach $\mathrm{Panse}$ angewendet. -

Zum Schlusse sei es mir gestattet, meinem hochverehrten Lehrer und Vorstand, Herrn Hofrat Prof. Dr. Politzer, meinen ergebensten Dank für die Überlassung des Krankenmaterials, sowie für die gütige Unterstützung auszuspreehen. Ebenso spreche ich meinen wärmsten Dank für die freundliche Unterstiutzung dem sehr geehrten Herrn Docenten Dr. G. Alexander aus. 